\title{
Características de los pacientes con crisis hipertensivas en Quemado de Güines, Villa Clara, Cuba
}

\section{Characteristics of patients with hypertensive crisis in Quemado de Güines, Villa Clara, Cuba}

\author{
Taimi Nereida Rodríguez Abrahantes*11 Gleudys Peralta Pérez ${ }^{2}$ Idalmis Veliz Vázquez ${ }^{3}$ Yadira Villavicencio \\ Martínez $^{2}$ Nercy Morales Morales ${ }^{2}$ \\ ${ }^{1}$ Licenciada en Enfermería. Master en Enfermedades Infecciosas. Profesora Auxiliar. Investigador, Cuba \\ ${ }^{2}$ Policlínico Mártires del 8 de abril. Quemado de Güines, Cuba \\ 3 Jefa departamento de Cuerpo guardia y Urgencias, Cuba \\ *taimirodriguez@infomed.sld.cu
}

DOI: https://doi.org/10.26871/killkana_salud.v4i3.687

\begin{abstract}
Resumen
Introducción: La hipertensión arterial (HTA) es la más común de las alteraciones que afectan a la salud de los individuos y las poblaciones en todas partes del mundo. Objetivo: determinar el comportamiento de los pacientes con crisis hipertensivas internados en el Área Intensiva del Policlínico Docente "Mártires 8 de abril". Métodos: se realizó una investigación descriptiva longitudinal retrospectiva, en el año 2018. La población estudio estuvo representada por la totalidad de los pacientes que acudieron a Emergencias con Hipertensión arterial. La muestra estuvo constituida por los 147 pacientes mayores de 15 años, de ambos sexos, que acudieron al Servicio de Urgencia del policlínico y requirieron ingreso en la Unidad Cuidados Intensivos con el diagnóstico de crisis hipertensiva. Los resultados se mostraron en tablas estadísticas de frecuencias absolutas y relativas. Resultados: predominó el ingreso en los grupos de edades mayores de 60 años, seguido del grupo de 50 y 59 años de edad. La principal modalidad de crisis se correspondió con las Urgencias Hipertensivas $(87,07 \%)$. El medicamento de mayor uso como tratamiento de la crisis fue el Captopril en tableta con un total del 93,87\%. El principal órgano diana afectado fue el corazón. Conclusiones: se destacó que las variables estudiadas en relación a ambas modalidades de las crisis hipertensivas, manifestaron resultados discretos en comparación a los hallados en otros estudios, con un predominio de las urgencias hipertensivas y el corazón como órgano diana más afectado.
\end{abstract}

Palabras clave: pacientes; crisis hipertensiva; área intensiva municipal.

\begin{abstract}
Introduction: Hypertension (HT) is the most common of the alterations that affect the health of individuals and populations in all parts of the world. Objective: to determine the behavior of patients with hypertensive crisis admitted to the Intensive Area of the Teaching Polyclinic "Mártires 8 de abril", 2018 Methods: a retrospective longitudinal descriptive investigation was carried out in 2018. The study population was represented by all the patients who attended the emergency room with 589 arterial hypertension. The sample consisted of 147 patients older than 15 years, of both sexes, who attended the Emergency Service of the polyclinic and required admission to the Intensive Care Unit with the diagnosis of hypertensive crisis. The results were shown in statistical tables of absolute and relative frequencies. Results: admission predominated in the age groups older than 60 years, followed by the group of 50 and 59 years of age. The main type of crisis corresponded to the Hypertensive Emergencies, (87.07\%). The most widely used medicine as a treatment for seizures was Captopril tablet with a total of $(93.87 \%)$. The main target organ affected was the heart. Conclusions: it was highlighted that the variables studied in relation to both modalities of hypertensive crises showed discrete results compared to those found in other studies, with a predominance of hypertensive emergencies and the heart as the most affected target organ.
\end{abstract}

Keywords: patients; hypertensive crisis; Municipal intensive área.

\section{Introducción}

La Hipertensión Arterial (HTA) ha sido vista a través de los años como "la más común de las condiciones que afectan al ser humano adulto, en toda latitud", ${ }_{1}$ su incidencia, sea directa o indirecta, sigue ocupando un lugar importante en los índices de mortalidad y morbilidad de la mayoría de los países del mundo contemporáneo. ${ }^{1,2}$

Aunque en la génesis de las afecciones cardiovasculares acontecen fenómenos multifactoriales, la HTA sigue siendo 
uno de los más importantes, tanto desde el punto de vista cualitativo como cuantitativo, al extremo de llegar a convertirse en el centro de atención de muchos investigadores y un importante objeto de estudio por parte de las estructuras sanitarias de todos los países. ${ }^{3}$

Enfermedades como la Enfermedad Cerebro Vascular (ECV), el Síndrome de Insuficiencia Coronaria (IC), la Arteriopatía Periférica, la Insuficiencia Cardiaca Congestiva y la Insuficiencia Renal Crónica, por tan solo mencionar las más conocidas, se presentan con una frecuencia entre dos y cuatro veces mayor en los pacientes hipertensos, independientemente de su edad y sexo, sin dejar de comentar que el riesgo de mortalidad en algunas de ellas es mucho mayor, cuando hablamos de un paciente hipertenso. Por otra parte la Insuficiencia Cardiaca y los síndromes coronarios se han venido incrementando en el último quinquenio en pacientes portadores de dicha condición. ${ }^{4,5}$

En el XXXVI Congreso Argentino de Cardiología, FAC2007, se señala que en el mundo se producen 5,1 millones de muertes al año por enfermedades cardiovasculares y que de ellas, el $62 \%$ están vinculadas al control subóptimo de la presión arterial. En ese cónclave se dio en llamar la HTA üna pandemia sin control". Mientras mejores resultados se obtengan en el control de la HTA menor será el riesgo cardiovascular del paciente y de la comunidad en su conjunto. 6,7

En Cuba se reportó oficialmente en el año 2017 un total de 22002 decesos en la población de 65 años y más por enfermedades del corazón las que de una forma u otra la Hipertensión Arterial jugó un papel determinante, con una cifra alarmante, 181.8 por cada 100000 habitantes en las edades de 50 a 64 años. ${ }^{8}$

Desde el año 2008 y hasta el 2010 algunos trabajos venían hablando de un 33,9\% de hipertensos nacionales, lo que equivalía a 2,5 millones de habitantes portadores de esta enfermedad, por lo que el tratamiento antihipertensivo ha cobrado un nuevo matiz, que va más allá de lograr solo el descenso de las cifras tensionales y se proyecta como principal meta, la reducción de la mortalidad y morbilidad de la esfera vascular asociada al exceso de presión. ${ }^{9,10}$

En el área de salud correspondiente al Policlínico Docente "Mártires 8 de abril", en el municipio de Quemado de Guines, se consultaron 589 pacientes en los meses de enero a diciembre de 2018 con HTA. Con el diagnóstico de crisis hipertensiva, 147 fueron ingresados en la sala de terapia Intensiva Municipal situada en el policlínico.

Interrogantes como: ¿Cuál fue la modalidad de crisis que prevaleció?; ¿Cómo respondieron a la terapéutica empleada?; ¿Cuál fue su destino final o principales complicaciones?; no siempre son abordadas en hojas de cargo y quedan al aire en muchas de las investigaciones revisadas, las cuáles hace énfasis en aspectos como factores de riesgo, datos estadísticos poblacionales, pero que no permiten un análisis palpable de la situación existente.

De acuerdo con lo anteriormente podemos plantear la interrogante científica que responde al problema científico de la investigación: ¿Cuál es el comportamiento de los pacientes diagnosticados con crisis hipertensiva ingresados en el servicio de terapia intensiva del Policlínico Mártires del 8 abril, Quemado de Guines, en el año 2018?

Todo lo anterior ha motivado a los autores a realizar esta investigación, que se sustenta, en el orden práctico, en lo explicado anteriormente y como objetivo persigue determinar el comportamiento de los pacientes con crisis hipertensivas ingresados en el área Intensiva del Policlínico Docente "Mártires 8 de abril de Quemado de Guines".

\section{Métodos}

Se realizó un estudio descriptivo longitudinal retrospectivo, durante el año 2018, en pacientes ingresados en el Área Intensiva Municipal del Policlínico Docente "Mártires 8 de abril", del municipio de Quemado de Guines.

La población estudio estuvo representada por los 147 pacientes mayores de 15 años, de ambos sexos, que acudieron al Servicio de Urgencia del policlínico y requirieron ingreso en la Unidad Cuidados Intensivos con el diagnóstico de crisis hipertensiva, coincidiendo con la muestra.

Para la recogida de información se diseñó una base de datos por los autores y avalada por expertos a partir de los libros de registro de este servicio y de historias clínicas. Además se obtuvieron datos en el departamento de archivo y estadística, así como también datos aportados por los propios pacientes y familiares en algunos casos. Se estudiaron variables como: edad (grupos de edades); sexo; estabilidad en el tratamiento antihipertensivo; tipo de crisis hipertensiva; toma de tensión arterial inicial y 30 minutos después de medicado; beneficios obtenidos con el tratamiento medicamentoso empleado; tipo de daño en órganos diana.

Para la clasificación de las crisis hipertensivas nos basamos en el Programa Nacional de HTA, el cual define las crisis hipertensivas como: un grupo de síndromes en los cuales una subida brusca de la presión arterial en individuos con HTA severa o moderada, se acompaña de manifestaciones reversibles o irreversibles de órganos diana, con una Presión Arterial (PA) diastólica mayor de 110 y se dividen en:

Emergencia Hipertensiva: cuando en presencia de una HTA severa se añaden disfunciones nuevas o agudas de órganos diana. La terapéutica debe administrase por la vía parenteral y los pacientes tienen criterio de ingreso en cuidados especiales o intensivos. Se incluyen aquí: encefalopatía hipertensiva, hemorragia intracraneal, angina de pecho inestable, infarto agudo del miocardio, insuficiencia ventricular aguda con edema pulmonar, aneurisma disecante de la aorta, eclampsia.

Urgencia hipertensiva: cuando hay subida tensional brusca en hipertensos moderados o severos, que no se acompaña de lesión de órgano diana inmediata, pero si lenta y progresiva, donde la PA debe reducirse en las siguientes 24 horas, por lo cual se utilizan fármacos orales. Aquí se incluyen el límite superior del Grado II, hipertensión con 
papiledema, lesión progresiva de órgano diana, hipertensión severa perioperatoria. ${ }^{11}$

Tabla 1: Operacionalizacion de las variables

\begin{tabular}{|c|c|c|c|}
\hline Variable & Tipo & Escala & Descripción \\
\hline Sexo & $\begin{array}{l}\text { Cualitativa } \\
\text { nominal } \\
\text { dicotómica }\end{array}$ & $\begin{array}{l}\text { Masculino } \\
\text { Femenino }\end{array}$ & $\begin{array}{l}\text { Según caracte- } \\
\text { rísticas biológi- } \\
\text { cas sexuales pri- } \\
\text { marias y secun- } \\
\text { darias. }\end{array}$ \\
\hline Edad & $\begin{array}{l}\text { Cuantitativa } \\
\text { continua }\end{array}$ & $\begin{array}{l}15-18 \\
19-29 \\
30-39 \\
40-49 \\
50-59 \\
60 \text { y mas }\end{array}$ & $\begin{array}{lr}\text { Según } & \text { edad } \\
\text { cumplida } & \text { en } \\
\text { el } & \text { momento } \\
\text { de } & \text { ocurrencia } \\
\text { del r } & \text { evento } \\
\text { cerebrovascular }\end{array}$ \\
\hline $\begin{array}{l}\text { Tipo de pre- } \\
\text { sentación }\end{array}$ & $\begin{array}{l}\text { Cualitativa } \\
\text { nominal } \\
\text { dicotómica }\end{array}$ & $\begin{array}{l}\text {-Urgencia } \\
\text { hipertensiva } \\
\text {-Emergencia } \\
\text { hipertensiva }\end{array}$ & $\begin{array}{l}\text { Elevación agu- } \\
\text { da de la presión } \\
\text { arterial capaz de } \\
\text { producir lesio- } \\
\text { nes en órganos } \\
\text { diana. Según la } \\
\text { presencia o no } \\
\text { de esta afecta- } \\
\text { ción, se clasifi- } \\
\text { ca en emergen- } \\
\text { cia hipertensiva } \\
\text { (EH) o urgen- } \\
\text { cia hipertensiva } \\
\text { (UH), respecti- } \\
\text { vamente. }\end{array}$ \\
\hline $\begin{array}{l}\text { Estabilidad en } \\
\text { el tratamiento }\end{array}$ & $\begin{array}{l}\text { Cualitativa } \\
\text { nominal } \\
\text { politómica }\end{array}$ & $\begin{array}{l}\text {-Siempre } \\
- \\
\text { Ocasionalmente } \\
\text {-Nunca }\end{array}$ & $\begin{array}{l}\text { Tiempo que } \\
\text { transcurre el } \\
\text { paciente con } \\
\text { cierto grado de } \\
\text { estabilidad } \\
\text { frente al } \\
\text { tratamiento } \\
\text { antihipertensivo } \\
\text { indicado. }\end{array}$ \\
\hline $\begin{array}{l}\text { Tipo de Antihi- } \\
\text { pertensivo uti- } \\
\text { lizado }\end{array}$ & $\begin{array}{l}\text { Cualitativa } \\
\text { nominal } \\
\text { politómica }\end{array}$ & $\begin{array}{l}\text {-Captopril } \\
\text { (tableta) } \\
\text {-Atenolol } \\
\text { (tableta) } \\
\text {-Nifedipina } \\
\text { (tableta) } \\
\text {-NTG (tableta) } \\
\text {-NTG } \\
\text { (ámpula) } \\
\end{array}$ & $\begin{array}{l}\text { Diversos } \\
\text { fármacos } \\
\text { utilizados en } \\
\text { medicina para } \\
\text { el tratamiento } \\
\text { de la hiperten- } \\
\text { sión.(reducción } \\
\text { de la PA) }\end{array}$ \\
\hline $\begin{array}{l}\text { Daño a Órga- } \\
\text { nos diana }\end{array}$ & $\begin{array}{l}\text { Cualitativa } \\
\text { nominal } \\
\text { politómica }\end{array}$ & $\begin{array}{l}\text {-Enfermedad } \\
\text { Cerebrovascu- } \\
\text { lar } \\
\text {-Encefalopatia } \\
\text { Hipertensiva } \\
\text {-Edema Agudo } \\
\text { del pulmón } \\
\text { - Insuficiencia } \\
\text { coronaria } \\
\text {-Infarto } \\
\text {-Arritmias } \\
\text { graves }\end{array}$ & $\begin{array}{l}\text { Lesión o daño } \\
\text { a órganos que } \\
\text { sufren daño de } \\
\text { forma secunda- } \\
\text { ria a la evolu- } \\
\text { ción de la enfer- } \\
\text { medad. }\end{array}$ \\
\hline
\end{tabular}

\subsection{Procesamiento de la información}

Los datos fueron procesados mediante paquete estadístico (SPSS) versión 10.0 y Microsoft Excel 2010 para Windows 7 y se presentaron en tablas estadísticas de frecuencias absolutas y relativas para su mejor comprensión.

\section{2 Ética de la Investigación}

En la presente investigación se cumple con los principios de la ética médica sobre todo el respeto y la autonomía, garantizando la confiabilidad del trabajo

\section{Resultados}

Como evidencia la tabla 2, predominó el grupo de edades de 60 años y más $(56,46 \%)$, seguida del grupo de edad entre 50 y 59 años $(25,85 \%)$, con predominio del sexo femenino.

Tabla 2: Pacientes con crisis hipertensivas según grupos de edades y sexo. Servicio de Terapia Intensiva. Policlínico. Quemado de Guines, 2018

\begin{tabular}{|l|l|l|l|l|l|l|}
\hline \multirow{2}{*}{$\begin{array}{c}\text { Grupos de edades } \\
\text { (años) }\end{array}$} & \multicolumn{2}{|c|}{ Masculino } & \multicolumn{2}{c|}{ Femenino } & \multicolumn{2}{c|}{ Total } \\
\cline { 2 - 7 } & No & $\%$ & No & $\%$ & No & $\%$ \\
\hline $15-18$ & 0 & 0 & 2 & 1.3 & 2 & 1.3 \\
\hline $19-29$ & 2 & 1.3 & 2 & 1.3 & 4 & 2.72 \\
\hline $30-39$ & 3 & 2.04 & 6 & 4.08 & 9 & 6.12 \\
\hline $40-49$ & 6 & 4.08 & 5 & 3.40 & 11 & 7.48 \\
\hline $50-59$ & 16 & 10.88 & 22 & 14.96 & 38 & 25.85 \\
\hline 60 y mas & 39 & 26.53 & 44 & 29.93 & 83 & 56.46 \\
\hline Total & 66 & 45.83 & 81 & 56.25 & 147 & 100.00 \\
\hline
\end{tabular}

Fuente: Modelo de recogida de datos

En la tabla 3 se distribuyeron los pacientes según la modalidad de crisis evidenciada, resultando que la mayoría de los ingresos se correspondieron con Ürgencias"(87,07\%), solo un $12.92 \%$ ingresaron por . Emergencias".

Tabla 3: Tipo de presentación de las crisis hipertensivas según sexo

\begin{tabular}{|l|l|c|c|c|c|c|}
\hline \multirow{2}{*}{ Tipo de presentación } & \multicolumn{2}{|c|}{ Masculino } & \multicolumn{2}{c|}{ Femenino } & \multicolumn{2}{c|}{ Total } \\
\cline { 2 - 8 } & No & $\%$ & No & $\%$ & No & $\%$ \\
\hline Urgencia & 58 & 39.45 & 70 & 47.61 & 128 & 87.07 \\
\hline Emergencia & 10 & 6.80 & 9 & 6.12 & 19 & 12.92 \\
\hline Total & 68 & 46.25 & 79 & 53.74 & 147 & 100.00 \\
\hline
\end{tabular}

Fuente: Modelo de recogida de datos

En la tabla 4 se analizó la estabilidad o adhesión al tratamiento por parte de los pacientes ingresados, obteniendo que el 59,18\% se medicara ocasionalmente, y el 30,01\% consumía habitualmente alguna modalidad de tratamiento.

Tabla 4: Tipo de presentación de las crisis hipertensivas según estabilidad en el tratamiento

\begin{tabular}{|l|l|c|c|c|c|c|}
\hline \multirow{2}{*}{$\begin{array}{c}\text { Estabilidad en el } \\
\text { tratamiento }\end{array}$} & \multicolumn{4}{|c|}{ HTA Grave } & \multicolumn{2}{c|}{ Total } \\
\cline { 2 - 7 } & \multicolumn{2}{|c|}{ Urgencia } & \multicolumn{2}{c|}{ Emergencia } & \multicolumn{2}{c|}{} \\
\cline { 2 - 7 } & No & $\%$ & No & $\%$ & No & $\%$ \\
\hline Siempre & 44 & 29.93 & 6 & 4.08 & 50 & 34.01 \\
\hline Ocasionalmente & 76 & 51.70 & 11 & 7.48 & 87 & 59.18 \\
\hline Nunca & 8 & 5.44 & 2 & 1.36 & 10 & 6.80 \\
\hline Total & 128 & 87.07 & 19 & 12.92 & 147 & 100.00 \\
\hline
\end{tabular}

Fuente: Modelo de recogida de datos 
El tratamiento más empleado en el manejo de estas crisis fue el Captopril, con un 93,87\% de los pacientes tratados, seguido de la Nitroglicerina en tableta $(74,82 \%)$, el atenolol fue efectivo en casi el $60 \%$ de los pacientes. (Tabla 5)

Tabla 5: Evolución de los pacientes según antihipertensivo utilizado

\begin{tabular}{|l|c|c|c|c|c|c|c|c|}
\hline \multirow{2}{*}{$\begin{array}{c}\text { Antihipertensivo } \\
\text { utilizado }\end{array}$} & \multicolumn{2}{|c|}{ Igual } & \multicolumn{2}{c|}{ Mejorado } & \multicolumn{2}{c|}{ Complicado } & \multicolumn{2}{c|}{ Total } \\
\cline { 2 - 10 } & No & $\%$ & No & $\%$ & No & $\%$ & No & $\%$ \\
\hline Captopril (tableta) & 86 & 58.50 & 50 & 34.01 & 2 & 1.36 & 138 & 93.87 \\
\hline Atenolol (tableta) & 16 & 10.88 & 70 & 47.01 & 2 & 1.36 & 88 & 59.83 \\
\hline Nifedipina (tableta) & 21 & 14.28 & 49 & 33.33 & 6 & 4.08 & 75 & 51.02 \\
\hline NTG (Tableta) & 39 & 26.53 & 62 & 42.17 & 9 & 6.12 & 110 & 74.82 \\
\hline NTG (ámpula) & 6 & 4.08 & 26 & 17.68 & 0 & 0 & 32 & 21.76 \\
\hline
\end{tabular}

Fuente: Modelo de recogida de datos

Los principales daños a órganos diana (DOD) se plasmaron en la tabla 6 , donde se observa que predominaron las arritmias graves $31,57 \%$, seguidas de la Insuficiencia Coronaria 26,31 \% y el edema Agudo Pulmonar (EAP) con $21,05 \%$.

Tabla 6: Pacientes con crisis hipertensivas según daño a órganos diana

\begin{tabular}{|l|l|l|}
\hline \multicolumn{1}{|c|}{ Daño a Órganos diana } & No & \multicolumn{1}{c|}{$\%$} \\
\hline Enfermedad Cerebrovascular & 3 & 15.78 \\
\hline Encefalopatía Hipertensiva & 1 & 5.26 \\
\hline Edema Agudo del pulmón & 4 & 21.05 \\
\hline Insuficiencia coronaria & 5 & 26.31 \\
\hline Infarto & 0 & 0 \\
\hline Arritmias graves & 6 & 31.57 \\
\hline Total & 19 & 100.00 \\
\hline
\end{tabular}

Fuente: Modelo de recogida de datos

\section{Discusión}

Los resultados en relación a la incidencia de las enfermedades hipertensivas en edades cada vez más tempranas, coloca a los autores en una posición de alerta pues contradice lo que ha sido reportado en algunos estudios en el área de las Américas. ${ }^{12}$

Ha sido reportado por varios autores que el sexo como factor de riesgo de la hipertensión arterial se ha señalado en una relación muy similar entre hombres y mujeres, quedando el hecho de mayor prevalencia en el sexo masculino como un tabú del pasado siglo, ante la emancipación de la fuerza femenil, quien lucha por compartir igualdad de derechos y hábitos con los hombres., ${ }^{13,14}$ datos estos no coincidentes con los resultados del estudio.

En relación al tipo de hipertensión grave, se constata que el número de pacientes cuya hipertensión ocasionó daños a órganos diana, se mantuvo en los pronósticos esperados, o sea los clasificándolos como . Emergencias Hipertensivas", lo cual viene a armonizar lo señalado por otros autores en los últimos quinquenios. ${ }^{15}$

Es válido señalar que la línea divisoria entre ambos términos, a pesar de estar conceptualmente bien definida, resulta prácticamente invisible en algunas ocasiones, ya bien por la gravedad del cuadro en cuestión, o bien por lo aparatoso del cuadro clínico, que no siempre es sinónimo de gravedad, y la interpretación del personal médico cuando declara una u otra modalidad en base a lo observado y al cortejo sintomático acompañante que difiere entre uno y otro paciente.

El incremento del número de estas crisis parece estar asociado al descuido, o a la no adhesión a un tratamiento hipotensor específico, como se recoge en el presente estudio, cuyos resultados coinciden con estudios realizados por autores nacionales. ${ }^{10,16}$ No obstante de que el Captopril en tableta fuese el medicamento más usado y que forma parte de los medicamentos de primera línea del stock de urgencias de cada área de salud, los mejores resultados se obtienen con el empleo de la NTG en infusión o en tabletas y con el uso de Corinfar (Nifedipino) en tableta, según estudios referenciados. ${ }^{16}$

Mucha y variada ha sido la controversia con respecto al uso del Nifedipino y la predilección por la presentación retart"de estos anticálcicos, de las cuales no disponemos, y aunque desde hace algún tiempo se maneja con discrepancia el uso del Nifedipino sublingual, por los descensos bruscos de la TA evidenciados en algunos sujetos, en este estudio se muestra que casi la totalidad de los pacientes tratados con esta droga, de forma controlada y bajo régimen estricto de observación, mejoraron en un corto periodo de tiempo luego de su administración, aseveran los autores del estudio.

Es importante decir que en el contexto de una crisis hipertensiva, el descenso indiscriminado de las cifras de tensión arterial puede ser tan nocivo como el alza en sí, destacan los autores del estudio.

En el período de tiempo comprendido, no existieron decesos en ninguna de las dos modalidades de crisis estudiadas, lo cual habla indirectamente de la eficacia y la importancia de la implementación de la estrategia de las Áreas Intensivas Municipales (AIM) en el manejo y alivio oportuno de situaciones graves que pueden llevar al traste la vida de un paciente en pocos minutos y fallecer mientras espera ser trasladado a un hospital o complicarse en el traslado. Las AIM y su acercamiento a la comunidad, han favorecido positivamente en estos indicadores de salud, que entre otras cosas, garantizan calidad de vida a cada uno de estos pacientes.

De manera concluyente se destacó que las variables estudiadas en relación a ambas modalidades de las crisis hipertensivas, manifestaron resultados discretos en comparación a los hallados en otros estudios, con un predominio 
de las urgencias hipertensivas y el corazón como órgano diana más afectado.

Se recomienda la propuesta por los autores de estrategias o acciones dirigidas a disminuir los factores predisponentes de esta urgencia médica y minimizar los ingresos en las unidades intensivas del área de salud municipal.

\section{Fuente de Financiamiento}

Este estudio es autofinanciado.

\section{Conflicto de Intereses}

No existen conflictos personales, profesionales, financieroso de otro tipo.

\section{Consentimiento Informado}

Los autores cuentan con el consentimiento informado de los pacientes para la investigación, la publicación del caso y sus imágenes.

\section{Referencias Bibliográficas}

1. Hackam DG, Khan NA, Hemmelgarn BR, Rabkin SW, Touyz RM, Campbell NR, et al. The 2010 Canadian Hypertension Education Program recommendations for the management of hypertension: part 2 -therapy. Can J Cardiol. 2014; 26: 249-58. [Citado: 25 de marzo de 2019]

2. Kaplan NM. Treatment of hypertension: drug therapy. In: Kaplan's Clinical Hypertension, 10th edition. Philadelphia, Lippincott Williams \& Wilkins, 2010, Cap. VI; pp 192-273. 5.

3. European Society of Hypertension and the European Society of Cardiology. Guidelines for Management of Arterial Hypertension. The Task Force for the Management of Arterial Hypertension. J Hyperten. 2017; 25:1105-87. [Citado: 14 de Octubre de 2018]

4. Ordúñez P, Pérez Flores E. Hospedales J. Más allá del ámbito clínico en el cuidado de la hipertensión arterial. Rev Panam Salud Pública. 2014; 28(4):311-18. [Citado: 25 de Abril de 2018]

5. Sellén Crombet J, Sellén Sanchén E, Barroso Pacheco L, Sellén Sánchez S. Evaluación y diagnóstico de la Hipertensión Arterial. Rev Cubana Invest Bioméd. [Internet]. 2015; 28(1). [Citado: 4 de enero de 2019] Disponible en: http://scielo.sld.cu/scielo.php?script=sci_arttext\& pid=S0864-03002009000100001\&lng=es .

6. Chobanian AV, Bakris GL, Black HR, Cushman WC, Green LA, Izzo JL, et al. The Seventh Report of the Joint National Committee on Prevention, Detection, Evaluation, and Treatment of High Blood Pressure: the JNC 7th report. JAMA. 2014; 289: 2560-72. [Citado: 4 de marzo de 2018]

7. Brent ME, Yumin Z, Neal Axon R. US trends in prevalence, awarensess, treatmen and control of hypertension.1988-2008. JAMA. 2015; 203(20):204350). [Citado: 15 de enero de 2019]
8. Ministerio de Salud Pública. Anuario Estadístico del 2011. Ciudad de la Habana. Cuba. MINSAP, 2012.

9. Dueñas A, Hernández O, Armas N, Sosa E, de la Noval R, Aguiar J. Manejo comunitario de la hipertensión arterial en el municipio Jagüey Grande. Matanzas. Rev Cubana Cardiol Cir Cardiovasc. 2015; 16(3):251-8. [Citado: 5 de agosto de 2018]

10. Alfonzo Guerra J, García Barreto D, Pérez Caballero D, Hernández Cueto M. Tratamiento Farmacológico. Cap. 14. En: Alfonzo Guerra JP. Hipertensión arterial en la atención primaria de salud. La Habana: Ed. Ciencias Médicas; 2014. p. 177-99.

11. Ministerio de Salud Pública. Guía Cubana para la Prevención, Diagnóstico y Tratamiento de la Hipertensión Arterial. Editorial Ciencias Médicas. Año 2008.

12. Frans H, Leenen H, Ernesto LS. Control rates of hypertension in North America. [Editorial]. Hipertensión. 2015; 56: 571. [Citado: 15 de octubre de 2018]

13. Achiong Estupiñán FJ, Olano Moreno M, Fong Ramírez E, Alfonso León JA, Achiong Alemañy M, Achiong Alemañy F. Intervención en hipertensos no controlados pertenecientes a la provincia de Matanzas en el año 2010. Rev Cub Hig Epidemiol [Internet]. 2016 [citado 8 Dic 2018]; 49(3). Disponible en: http://scielo.sld.cu/scielo.php?script=sci_arttext\& pid=S1561-30032011000300006\&lng=es.

4. Niiranen TJ, Hänninen MR, Johansson J, Reunanen A, Jula AM. Home-measured blood pressure is a stronger predictor of cardiovascular risk than office blood pressure: the Finn-Home study. Hipertensión 2015; 55: 1346-1351. E-Ib. [citado: 8 Noviembre de 2018]

15. Ibáñez-Torales. Hipertensión Arterial: emergencias y urgencias. [Consulta: 20 mayo 2011]. <http: //www.intramed.net/sitios/librovirtual1/pdf/librovirtual1_ 10.pdf>

16. Bharati V, Mittal MD, Ajay K, Singh MB. Hypertension in the Developing World: Challenges and Opportunities. Am J Kidney Dis. 2015; 55:590-8. [Citado: 22 de abril de 2019]

Recibido: 6 de abril de 2020

Aceptado: 15 de agosto de 2020 
